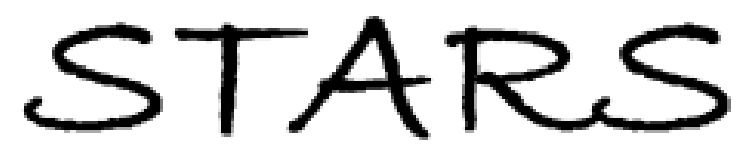

University of Central Florida

STARS

$1-1-2011$

\title{
Phase, grain structure, stress, and resistivity of sputter-deposited tungsten films
}

Dooho Choi

Bincheng Wang

Suk Chung

Xuan Liu

Amith Darbal

See next page for additional authors

Find similar works at: https://stars.library.ucf.edu/facultybib2010

University of Central Florida Libraries http://library.ucf.edu

This Article is brought to you for free and open access by the Faculty Bibliography at STARS. It has been accepted for inclusion in Faculty Bibliography 2010s by an authorized administrator of STARS. For more information, please contactSTARS@ucf.edu.

\section{Recommended Citation}

Choi, Dooho; Wang, Bincheng; Chung, Suk; Liu, Xuan; Darbal, Amith; Wise, Adam; Nuhfer, Noel T.; Barmak, Katayun; Warren, Andrew P.; Coffey, Kevin R.; and Toney, Michael F., "Phase, grain structure, stress, and resistivity of sputter-deposited tungsten films" (2011). Faculty Bibliography 2010s. 1179.

https://stars.library.ucf.edu/facultybib2010/1179

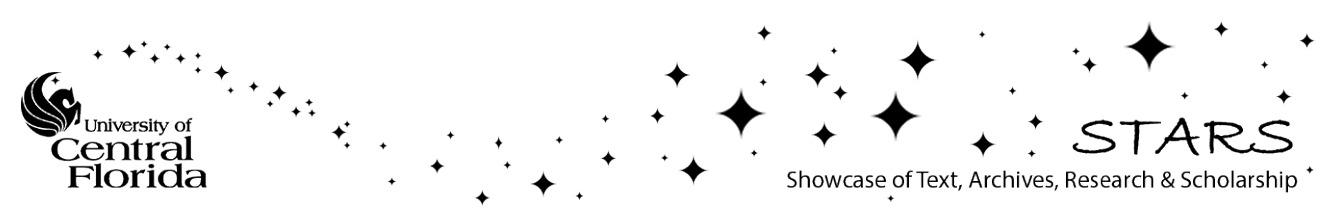




\section{Authors}

Dooho Choi, Bincheng Wang, Suk Chung, Xuan Liu, Amith Darbal, Adam Wise, Noel T. Nuhfer, Katayun

Barmak, Andrew P. Warren, Kevin R. Coffey, and Michael F. Toney 


\section{Phase, grain structure, stress, and resistivity of sputter-deposited tungsten films}

Dooho Choi, Bincheng Wang, Suk Chung, Xuan Liu, Amith Darbal, Adam Wise, Noel T. Nuhfer, Katayun Barmak, Andrew P. Warren, Kevin R. Coffey, and Michael F. Toney

Citation: Journal of Vacuum Science \& Technology A 29, 051512 (2011); doi: 10.1116/1.3622619

View online: https://doi.org/10.1116/1.3622619

View Table of Contents: https://avs.scitation.org/toc/jva/29/5

Published by the American Vacuum Society

\section{ARTICLES YOU MAY BE INTERESTED IN}

Phase transformation of thin sputter-deposited tungsten films at room temperature Journal of Vacuum Science \& Technology B: Microelectronics and Nanometer Structures Processing, Measurement, and Phenomena 20, 2047 (2002); https://doi.org/10.1116/1.1506905

Microstructure, growth, resistivity, and stresses in thin tungsten films deposited by rf sputtering Journal of Applied Physics 44, 2545 (1973); https://doi.org/10.1063/1.1662611

Residual stress, microstructure, and structure of tungsten thin films deposited by magnetron sputtering Journal of Applied Physics 87, 177 (2000); https://doi.org/10.1063/1.371841

Structure and stability of sputter deposited beta-tungsten thin films Applied Physics Letters 64, 3231 (1994); https://doi.org/10.1063/1.111318

Beta $(\beta)$ tungsten thin films: Structure, electron transport, and giant spin Hall effect Applied Physics Letters 106, 182403 (2015); https://doi.org/10.1063/1.4919867

Stress, strain, and microstructure in thin tungsten films deposited by dc magnetron sputtering Journal of Applied Physics 74, 988 (1993); https://doi.org/10.1063/1.354842

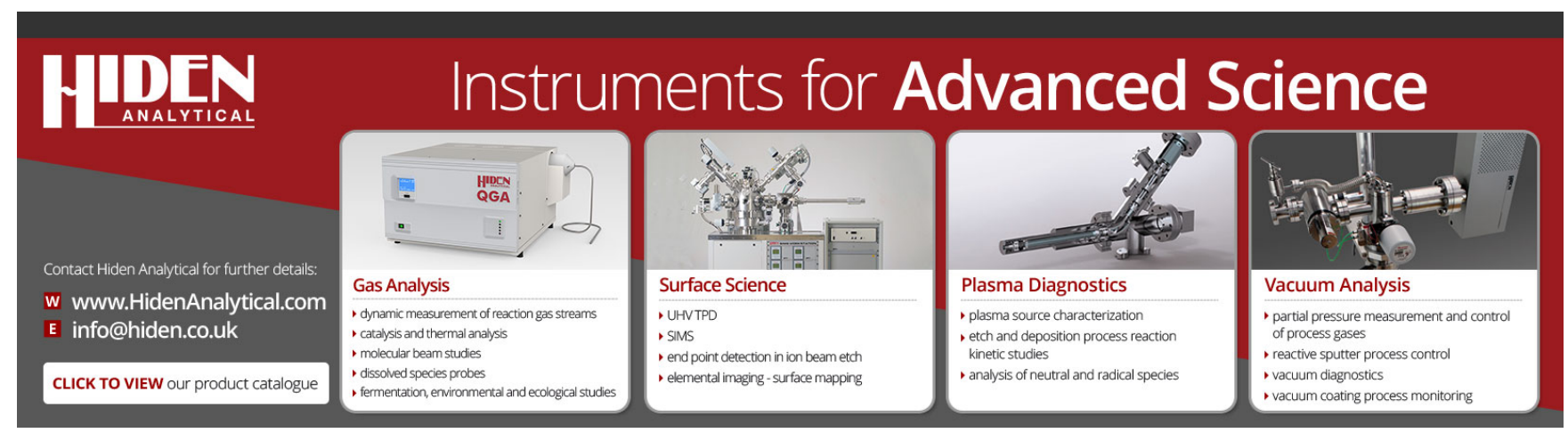




\title{
Phase, grain structure, stress, and resistivity of sputter-deposited tungsten films
}

\author{
Dooho Choi, Bincheng Wang, Suk Chung, Xuan Liu, Amith Darbal, Adam Wise, \\ Noel T. Nuhfer, and Katayun Barmak ${ }^{\text {a) }}$ \\ Materials Research Science and Engineering Center and the Department of Materials Science and \\ Engineering, Carnegie Mellon University, 5000 Forbes Avenue, Pittsburgh, Pennsylvania 15213
}

Andrew P. Warren and Kevin R. Coffey

Advanced Materials Processing and Analysis Center, University of Central Florida, 4000 Central Florida Boulevard, Orlando, Florida 32816

Michael F. Toney

Stanford Synchrotron Radiation Lightsource, 2575 Sand Hill Road, Menlo Park, California 94025

(Received 21 April 2011; accepted 15 July 2011; published 15 August 2011)

\begin{abstract}
Sputter-deposited W films with nominal thicknesses between 5 and $180 \mathrm{~nm}$ were prepared by varying the base pressure prior to film deposition and by including or not including sputtered $\mathrm{SiO}_{2}$ encapsulation layers. X-ray and electron diffraction studies showed that single phase, polycrystalline $\alpha-\mathrm{W}$ could be achieved in as-deposited films as thin as $5 \mathrm{~nm}$. The stress state in the as-deposited films was found to be inhomogeneous. Annealing resulted in stress relaxation and reduction of resistivity for all films, except the thinnest, unencapsulated film, which agglomerated. In-plane film grain sizes measured for a subset of the annealed films with thicknesses between 5 and $180 \mathrm{~nm}$ surprisingly showed a near constant value $(101-116 \mathrm{~nm})$, independent of film thickness. Thick-film $(\geq 120 \mathrm{~nm})$ resistivity values as low as $8.6 \mu \Omega \mathrm{cm}$ at $301 \mathrm{~K}$ were obtained after annealing at $850^{\circ} \mathrm{C}$ for $2 \mathrm{~h}$. Film resistivities were found to increase with decreasing film thicknesses below $120 \mathrm{~nm}$, even for films which are fully A2 $\alpha-\mathrm{W}$ with no metastable, A15 $\beta-\mathrm{W}$ evident. (C) 2011 American Vacuum Society. [DOI: 10.1116/1.3622619]
\end{abstract}

\section{INTRODUCTION}

$\mathrm{Cu}$, the current interconnect material of choice for semiconductor metallization, suffers from two shortcomings. The first is the resistivity size effect, namely, the sharp rise in electrical resistivity when sample structural dimensions (thicknesses in case of films, and line heights and widths in case of interconnects) are reduced towards and then below its relatively large electron mean free path (EMFP) of $39 \mathrm{~nm}$ at room temperature. ${ }^{1-4}$ This resistivity rise has been identified as a Grand Challenge Problem by the International Technology Roadmap for Semiconductors (ITRS). ${ }^{5}$ The second shortcoming is the degraded reliability of $\mathrm{Cu}$ as device operating temperatures and current densities increase with each technology node. ${ }^{6-8}$ These two shortcomings of $\mathrm{Cu}$ have prompted consideration of alternate interconnect materials and schemes over the past few years. ${ }^{9-13} \mathrm{~W}$ is one such candidate material for consideration, for reasons discussed in the following.

The upper limit to the EMFP of $\mathrm{W}$ is reported as $10 \mathrm{~nm}$ (Ref. 14) and may be as low as $2 \mathrm{~nm} .{ }^{15}$ Therefore, despite the fact that the room-temperature ( $293 \mathrm{~K}$ ) bulk resistivity of $\mathrm{W}$ at $5.3 \mu \Omega \mathrm{cm}$ (Ref. 16) is more than three times higher than that for $\mathrm{Cu}$ at $1.7 \mu \Omega \mathrm{cm}$, the resistivity of $\mathrm{W}$ is predicted to cross below that for $\mathrm{Cu}$ at linewidths below 25 $\mathrm{nm} .{ }^{17} \mathrm{In}$ addition, the higher melting temperature of $\mathrm{W}$ at

${ }^{a)}$ Electronic mail: Katayun@andrew.cmu.edu
$3695 \mathrm{~K}$ (more than twice that of $\mathrm{Cu}$ at $1358 \mathrm{~K}$ ) should result in dramatically improved interconnect reliability, qualitatively similar to the improvement observed when $\mathrm{Cu}$ was introduced to replace $\mathrm{Al}$ interconnects. ${ }^{18}$ For an operating temperature of $413 \mathrm{~K}\left(140^{\circ} \mathrm{C}\right), \mathrm{Cu}$ is at slightly more than $30 \%$ of its melting temperature, and degradation (electromigration, stress voiding, etc.) by the thermally activated mechanisms of surface and grain boundary diffusion occurs. ${ }^{18}$ At this same temperature, W will be at only $11 \%$ of its melting temperature, which should lead to a significant reduction of the rates of these thermally activated degradation mechanisms.

$\mathrm{W}$ also offers the possibility of eliminating the bilayer $\mathrm{TaN} / \mathrm{Ta}$ diffusion barrier-adhesion layers currently used for $\mathrm{Cu}{ }^{19,20}$ Further, since $\mathrm{W}$ has been used as plugs and vias in current complementary metal-oxide-semiconductor structures, its compatibility with interlayer dielectric materials and back-end-of-line processes is partly established. ${ }^{21}$

In summary, the expectations that make $\mathrm{W}$ a worthwhile candidate material for study as an alternate conductor to $\mathrm{Cu}$ are a reduced size effect, greater reliability, and the potential for eliminating diffusion barrier-adhesion layers. The challenge faced for $\mathrm{W}$ as an interconnect metal is obtaining the $5.3 \mu \Omega \mathrm{cm}$ bulk resistivity in polycrystalline films. For example, the reported resistivities for $\mathrm{W}$ films deposited by chemical vapor deposition (CVD) are typically greater than $8 \mu \Omega \mathrm{cm},{ }^{22}$ and for $\mathrm{W}$ films deposited by sputtering they are greater than $10 \mu \Omega \mathrm{cm}^{14}$ These higher resistivity values 
result in part from impurities in the film inherited from the source material and/or incorporated during film fabrication. ${ }^{14,22-28}$ In addition, $\mathrm{W}$ can exist in the form of a high-resistivity metastable A15 $\beta$ phase. The reported bulk resistivity for this phase is in the range of $150-350 \mu \Omega \mathrm{cm}$; therefore, even small quantities of $\beta$-W in the films can result in high film resistivities.

In this work, we examine the impact of the base pressure prior to film deposition, the film thickness (5-180 nm, nominal), the presence or absence of encapsulation layers of $\mathrm{SiO}_{2}$, and postdeposition annealing on the formation of the $\alpha$ - and $\beta$-W phases and on film resistivity. Grain structure and stress are also assessed. We show that single phase $\alpha-\mathrm{W}$ films can be obtained in as-deposited films as thin as $5 \mathrm{~nm}$ and a resistivity of $8.6 \mu \Omega \mathrm{cm}$ can be achieved for annealed films $\geq 120 \mathrm{~nm}$ thick. This thick-film resistivity value is lower than previously reported values for sputtered $\mathrm{W}$ films, ${ }^{24,25}$ and suggests that a further reduction of resistivity may be achievable by, for example, using higher purity sputtering targets, thereby encouraging continued investigation of $\mathrm{W}$ as a potential candidate metal for future interconnects.

\section{EXPERIMENT}

Three separate sets of $\mathrm{W}$ films, comprising 18 films (A20C180, Table I) were prepared for study. The films were deposited onto $75 \mathrm{~mm}$ diameter Si (100) wafers that had 100 $\mathrm{nm}$ of thermally grown oxide. Following our work on $\mathrm{Cu}$ films, ${ }^{3}$ two of the three sets of $\mathrm{W}$ films (groups A and B) were prepared as encapsulated films in the form of $\mathrm{Si} / 100$ $\mathrm{nm}$ thermal oxide/20 nm sputtered $\mathrm{SiO}_{2} / \mathrm{W} / 20 \mathrm{~nm}$ sputtered $\mathrm{SiO}_{2}$ stack. The sputtered $\mathrm{SiO}_{2}$ encapsulation provided for identical top and bottom electron scattering surfaces and minimized void formation during subsequent annealing. ${ }^{3}$ For one of the two encapsulated sets of $\mathrm{W}$ films, the base pressure of the chamber prior to deposition was $2 \times 10^{-9}$ Torr, and for the other set, it was $5 \times 10^{-7}$ Torr. The lower base pressure of $2 \times 10^{-9}$ Torr was achieved by baking the chamber prior to film deposition. The third set of $\mathrm{W}$ films (group C) had no encapsulating layers of sputtered $\mathrm{SiO}_{2}$. For these films, W was deposited directly on the oxidized Si wafer. The base pressure of the chamber prior to deposition of this unencapsulated set was $5 \times 10^{-7}$ Torr.

The $\mathrm{W}$ layers were deposited by dc sputtering from a 99.95\% pure $\mathrm{W}$ target, and the $\mathrm{SiO}_{2}$ layers were deposited by $\mathrm{RF}$ magnetron sputtering from a $99.995 \%$ pure $\mathrm{SiO}_{2}$ target. For all three sets of films, the sputtering gas was 99.999\% pure Ar gas that was additionally purified by passing through a purifier (SPG-MC50-902F by Fulcrum Technologies, Inc) prior to its introduction into the chamber. The sputtering pressure was 3 mTorr and the Ar flow rate was 20 $\mathrm{sccm}$.

The thicknesses of four films (B20, B30, B40, and B60, Table I) were measured using $\mathrm{X}$-ray reflectivity (XRR). The XRR patterns were obtained on the PANalytical PW 3040/60 instrument. Using these measured thicknesses and the total deposition time, the deposition rate was calculated at $1.4 \AA / \mathrm{s}$. This rate was then used to calculate the thicknesses of all the remaining films. The nominal and measured or calculated film thicknesses are given in Table I.

The wafers were sectioned into samples of $1.5 \mathrm{~cm} \times 1.5$ $\mathrm{cm}$ in size for further processing and characterization. Annealing was carried out ex situ in a quartz tube furnace for $2 \mathrm{~h}$ at $400{ }^{\circ} \mathrm{C}$ (subset B20-B180) or at $850^{\circ} \mathrm{C}$ (all films) in an $\mathrm{Ar}-4 \% \mathrm{H}_{2}$ ambient. In order to minimize impurity incorporation during annealing, the tube was evacuated

TABLE I. Film identification, base pressure prior to film deposition, film thickness, and film resistivities in the as-deposited and annealed states. The number of grains measured and the grain size for a subset of the annealed films are also given. Films were annealed at $850{ }^{\circ} \mathrm{C}$ for $2 \mathrm{~h}$.

\begin{tabular}{|c|c|c|c|c|c|c|c|}
\hline \multirow[b]{2}{*}{ Film id. } & \multirow[b]{2}{*}{ Base pressure (Torr) } & \multirow[b]{2}{*}{$\mathrm{SiO}_{2}$ encapsulation } & \multirow[b]{2}{*}{ Film thickness (nm) } & \multicolumn{2}{|c|}{ Resistivity at $301 \mathrm{~K}(\mu \Omega-\mathrm{cm})$} & \multirow[b]{2}{*}{ No. of grains measured } & \multirow[b]{2}{*}{ Grain Size (nm) } \\
\hline & & & & As-deposited & $850^{\circ} \mathrm{C}, 2 \mathrm{~h}$ & & \\
\hline A 20 & $5 \times 10^{-7}$ & Yes & 18.9 & 137.8 & 17.0 & & \\
\hline A180 & & & 180.1 & 11.6 & 10.2 & & \\
\hline B5 & $2 \times 10^{-9}$ & Yes & 5.1 & 116.6 & 21.8 & & \\
\hline B10 & & & 9.3 & 29.8 & 18.5 & & \\
\hline B20 & & & 18.9 & 18.6 & 16.5 & 1048 & $102 \pm 6$ \\
\hline B30 & & & 28.3 & 14.6 & 13.5 & 1088 & $106 \pm 6$ \\
\hline B40 & & & 42.5 & 12.8 & 12.0 & 1103 & $101 \pm 6$ \\
\hline B60 & & & 60.7 & 12.1 & 11.4 & 1445 & $111 \pm 5$ \\
\hline B120 & & & 122.9 & 11.1 & 10.3 & 1019 & $109 \pm 6$ \\
\hline B 180 & & & 180.1 & 10.7 & 10.0 & 1064 & $116 \pm 6$ \\
\hline C5 & $5 \times 10^{-7}$ & No & 5.1 & 31.4 & a & & \\
\hline $\mathrm{C} 10$ & & & 9.5 & 19.1 & 15.9 & & \\
\hline $\mathrm{C} 20$ & & & 19.0 & 13.3 & 12.5 & & \\
\hline $\mathrm{C} 30$ & & & 28.5 & 12.4 & 10.8 & & \\
\hline $\mathrm{C} 40$ & & & 43.2 & 10.9 & 9.8 & & \\
\hline C60 & & & 61.8 & 10.5 & 9.3 & & \\
\hline C120 & & & 124.4 & 9.5 & 8.6 & & \\
\hline C180 & & & 182.3 & 9.5 & 8.6 & & \\
\hline
\end{tabular}

${ }^{\mathrm{a}}$ Film agglomerated during annealing. 
before the furnace temperature was raised and $\mathrm{Ar} / 4 \% \mathrm{H}_{2}$ was introduced. For phase identification, $\theta-2 \theta$ x-ray diffraction (XRD) scans were performed on the PANalytical PW 3040/60 diffractometer using $\mathrm{Cu} K \alpha$ radiation. The samples were omega-tilted by $6.5^{\circ}$ in order to suppress the strong $\mathrm{Si}$ substrate peak $\left(2 \theta \sim 69.2^{\circ}\right)$, without significantly affecting the intensity of the $\mathrm{W}$ peaks. All the XRD scans were performed immediately after film deposition since room-temperature transformation of $\beta$-W to $\alpha-\mathrm{W}$ has been noted by other researchers. ${ }^{14}$ A small drop in resistivity was observed over approximately 2 weeks for a $20 \mathrm{~nm}$ film (film A20), but not for thicker films.

The stress state of films B20-B180 was determined by using both the out-of-plane $\theta-2 \theta$ scans described earlier and in-plane $\theta-2 \theta$ scans performed at a sample tilt of $87^{\circ}$ relative to substrate normal. The magnitude of the in-plane stress in the $\mathrm{W}$ films was assessed by perpendicular strains caused by Poisson expansion or contraction. These strains were determined using the (110) XRD peak of $\alpha$-W in the out-of-plane scans. If the peaks were asymmetric (due to an inhomogeneous stress state in the film), they were deconvoluted into two subpeaks, following the work by Noyan and Shaw. ${ }^{30}$ The peak position, full width at half maximum, area, and percentage of Lorentzian and Gaussian components for the two subpeaks were optimized in the fits.

Phases and microstructure of selected as-deposited films were characterized by transmission electron microscopy (TEM) examination in a JEOL 2000EX. The TEM samples were prepared by mechanical polishing, dimpling, and ion milling to electron transparency. Mean grain size of a subset of annealed samples was obtained via conical dark-field imaging of plan-view samples using the hardware-software setup Automated Crystallography in the TEM (ACT). ${ }^{31,32}$ Approximately 1000 grains were measured for each sample (Table I). The reported grain size in Table I is the equivalent circle diameter of the mean area, i.e., grain size $=\sqrt{\frac{4\langle A\rangle}{\pi}}$, where $\langle\mathrm{A}\rangle$ is the mean area. The errors on these diameters are quoted as $\pm 2 \sigma$ values at a confidence level of $95 \%$ for the given grain population. ${ }^{33}$

The sheet resistance of the films was measured at room temperature (typically $301 \mathrm{~K}$ in our laboratory) using the Van der Pauw method ${ }^{34}$ and an Agilent 34420A Nano Volt/Micro Ohm Meter. The two pairs of contact pins of the dipping probe pressed onto the samples readily penetrated the $20 \mathrm{~nm}$ of the sputtered $\mathrm{SiO}_{2}$ overlayer (for groups $\mathrm{A}$ and B) to form an electrical contact to the $\mathrm{W}$ films.

\section{RESULTS AND DISCUSSION}

For films A20-C180, Table I gives the base pressure prior to film deposition, the presence or absence of the $\mathrm{SiO}_{2}$ encapsulation layers, and the measured thickness of the $\mathrm{W}$ layer. The room-temperature resistivities of the films in the as-deposited state and after annealing at $850{ }^{\circ} \mathrm{C}$ for 2 $\mathrm{h}$ are also listed in Table I. The grain sizes for a subset of the annealed films and the number of grains measured for each film are given in Table I. Annealing at $400^{\circ} \mathrm{C}(\mathrm{B} 20-$
B180) resulted in relatively small changes compared to the annealing at $850{ }^{\circ} \mathrm{C}$, and therefore, these results are not reported.

\section{A. Phases}

Figure 1 presents the XRD patterns for films A20, B20, and C20 obtained immediately after deposition. Film A20, for which the base pressure prior to deposition was $5 \times 10^{-7}$ Torr and for which $\mathrm{SiO}_{2}$ encapsulation layers were present, was $\beta$-W. In contrast, film B20, which differs from film A20 in that the base pressure prior to deposition was lower at $2 \times 10^{-9}$ Torr, and film $\mathrm{C} 20$, which has no $\mathrm{SiO}_{2}$ encapsulation layers, are both $\alpha-\mathrm{W}$. Figure 1 also presents the XRD patterns for A180, B180, and C180. All three films are $\alpha-\mathrm{W}$ regardless of the base pressure prior to deposition or the $a b-$ sence or presence of the $\mathrm{SiO}_{2}$ encapsulation layers. The $\alpha-\mathrm{W}$ films were either untextured or only very weakly (110) textured.

Figures 2(a)-2(d) show the plan-view dark-field TEM images with the selected area diffraction patterns (SADPs) as insets for films B5,C5, B10, and C10. As seen in Fig. 2(a), film $\mathrm{B} 5$, encapsulated with $\mathrm{SiO}_{2}$ and prepared with the base pressure of $2 \times 10^{-9}$ Torr, contains coarse $\alpha-\mathrm{W}$ grains in a matrix of fine $\beta-\mathrm{W}$ grains. The large $\alpha-\mathrm{W}$ grains give rise to the bright diffraction spots in the SADP, whereas the fine grains of $\beta-\mathrm{W}$ result in the diffuse rings. Figures $2(\mathrm{~b})-2(\mathrm{~d})$ show that film $\mathrm{C} 5$, and films B10 and $\mathrm{C} 10$ are single phase, coarse-grained $\alpha-\mathrm{W}$. Film B10 was deposited under similar conditions to film B5 but has twice the nominal thickness. Film $\mathrm{C} 5$ has the same nominal thickness as film B5, but lacks the encapsulation layers. Film C10 also lacks the encapsulation layers and has the same nominal thickness as film B10.

The presence of $\beta$-W in film B5 was further confirmed by $\mathrm{XRD}$, as seen in the enlarged section of the $\theta-2 \theta$ pattern shown in Fig. 3(a). For films C5, B10, and C10, the enlarged sections of the $\theta-2 \theta$ XRD patterns in Figs. 3(b)-3(d) show

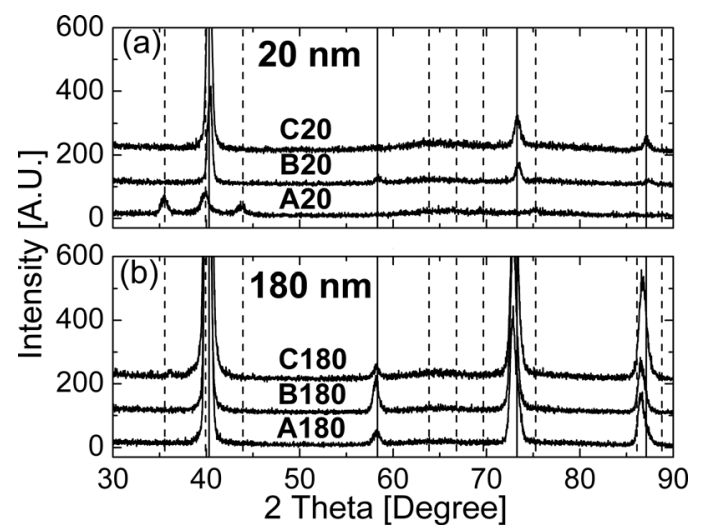

FIG. 1. $\theta-2 \theta$ XRD patterns for the as-deposited films A20, A180, B20, B180, C20, and C180 obtained immediately following deposition. The measured film thicknesses are given in Table I. The solid and dotted vertical lines mark the positions of the Bragg peaks for $\alpha-\mathrm{W}$ and $\beta$-W structures, respectively. For $\alpha-W$, the four Bragg peaks are, from left to right, (110), (200), (211), and (220). For $\beta$-W, the nine Bragg peaks are, from left to right, (200), (210), (211), (222), (320), (321), (400), (420), and (421). 

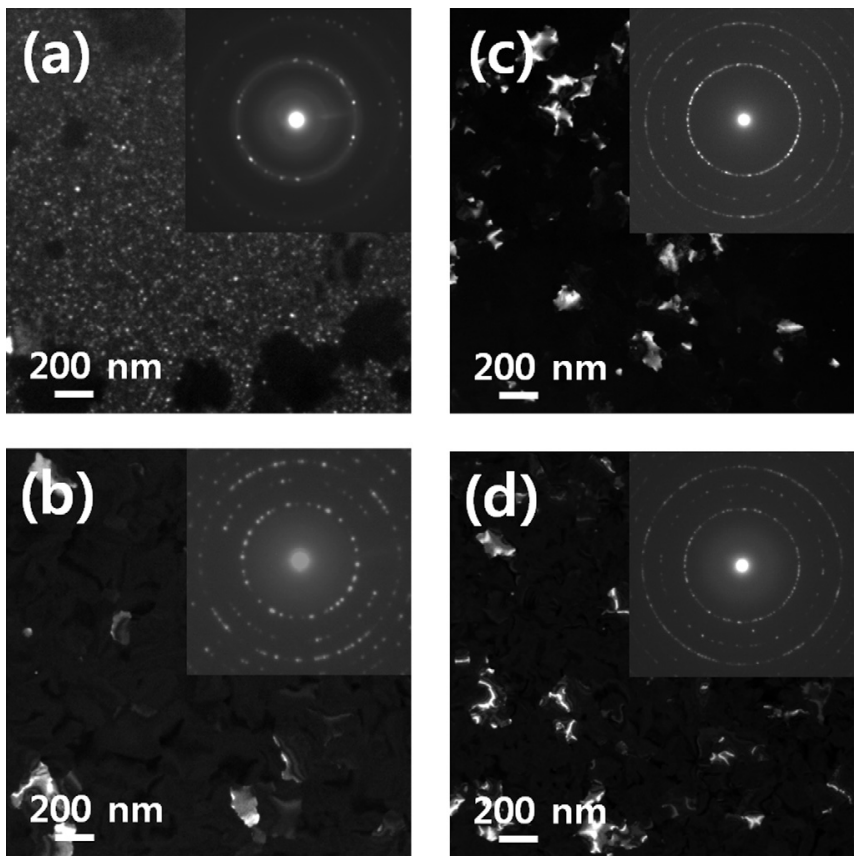

FIG. 2. Dark-field transmission electron micrographs for the nominally 5nm-thick films B5 (a) and C5 (b) and the nominally 10-nm-thick films B10 (c) and $\mathrm{C} 10(\mathrm{~d})$. The selected area diffraction patterns are shown as insets in the upper right.

them to be $\alpha-W$, in agreement with the SADPs in Figs. 2(b)2(d). From these results, it is concluded that the formation of $\alpha-\mathrm{W}$ in the thinner as-deposited films is promoted by either using a lower base pressure prior to film deposition or by omitting the $\mathrm{SiO}_{2}$ encapsulation layers. However, the latter is more effective than the former, allowing us to obtain single phase $\alpha-\mathrm{W}$ in an unencapsulated, 5-nm-thick film in the as-deposited state (film C5).

\section{B. Grain structure}

TEM examination of plan-view samples of films with nominal thicknesses of $5 \mathrm{~nm}$ (B5, C5), $10 \mathrm{~nm}$ (B10, C10), 20 $\mathrm{nm}$ (B20, C20), and $180 \mathrm{~nm}$ (B180, C180) in the as-depos-

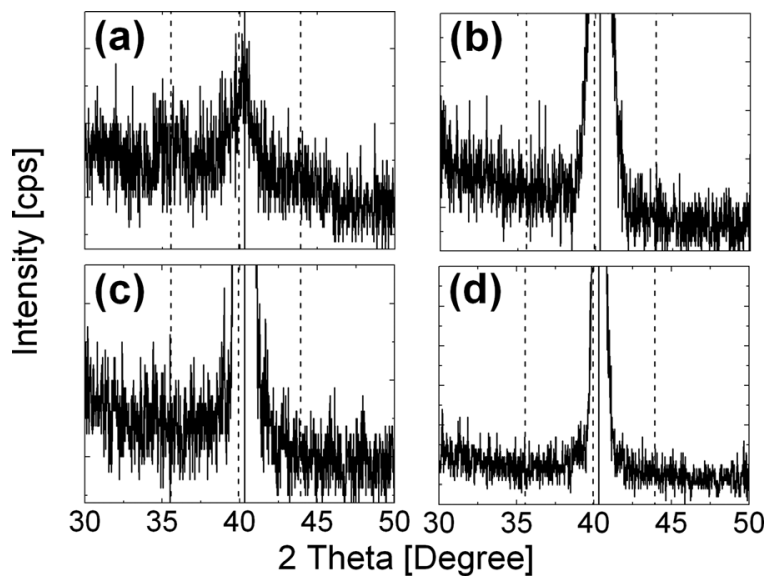

FIG. 3. Sections of the $\theta-2 \theta$ XRD patterns for films B5, B10, C5, and C10. The solid vertical lines in the patterns correspond to the (110) reflection of $\alpha-\mathrm{W}$, while the dotted vertical lines correspond in order from left to right to the (110), (200), and (211) reflections of $\beta$-W.

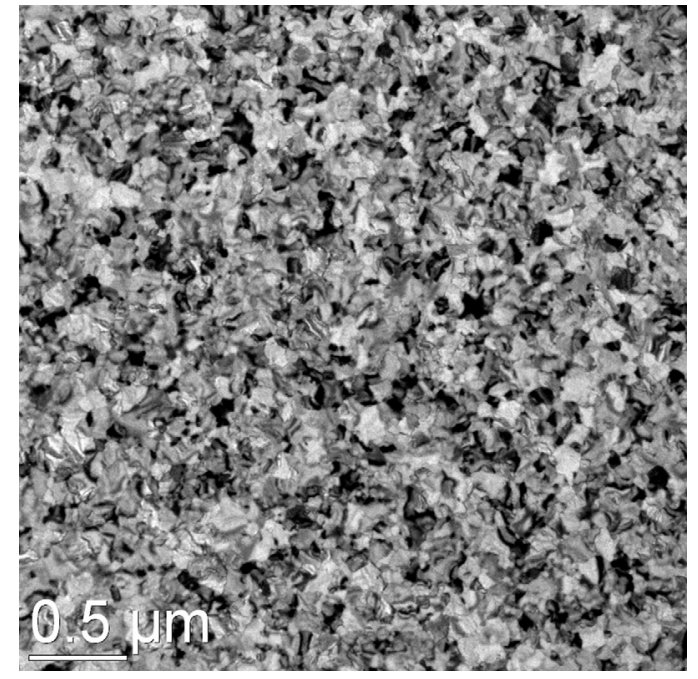

FIG. 4. Plan-view electron phase contrast TEM image of film B20 in the asdeposited state. The phase contrast image was formed by using a large enough objective aperture to include both the transmitted beam and some of the diffracted beams.

ited state showed them to be polycrystalline and void-free. As an example, the electron wave phase contrast image for film B20 is shown in Fig. 4. Dark-field cross-sectional TEM studies of as-deposited films B20, B180, and C180 showed the grain structure to be columnar. The cross-sectional image of film B180 in the as-deposited state is shown in Fig. 5 as an example.

The in-plane grain sizes measured for a subset of the $850^{\circ} \mathrm{C}$ annealed films (B20-B180) are given in Table I. Grain sizes for these films, which have nominal thicknesses between 20 and $180 \mathrm{~nm}$, are in the range of 101-116 nm; nearly independent of film thickness. One possible explanation for this surprising lack of dependence of $\alpha-\mathrm{W}$ grain size on film thickness is that the grain size of this phase is determined by the transformation of $\beta-\mathrm{W}$ to $\alpha-\mathrm{W}$ in the early stages of film deposition. In support of this explanation, see Fig. 2(a) for as-deposited film B5 where the size of the $\alpha-W$ grains in the matrix of $\beta$-W is in the $100 \mathrm{~nm}$ range. During the remainder of film deposition and the subsequent annealing, grain boundary mobility in $\alpha-\mathrm{W}$ is too low to result in grain coarsening. Thus, once the $\beta$ to $\alpha$ transformation is complete, the lateral grain size remains essentially unchanged as the film thickens during deposition or is annealed after deposition. ${ }^{35,36}$

\section{Film stress}

Figure 6(a) shows the enlarged sections of the $\alpha-(110)$ XRD peak for out-of-plane scans for six as-deposited films

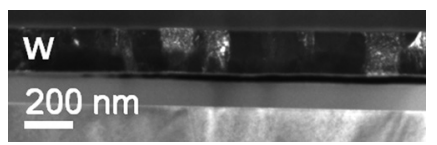

FIG. 5. Cross-sectional dark-field transmission electron micrograph of film B180 in the as-deposited state. This image was formed by using the (110) reflection. 
(B20, C20, B30, C30, B180, and C180). The peaks for films B20, B30, B180 (encapsulated, base pressure of $2 \times 10^{-9}$ Torr) are given in the top row, and those for films C20, C30, and $\mathrm{C} 180$ (not encapsulated, base pressure of $5 \times 10^{-7}$ Torr) are given in the bottom row. Results only for these six films are shown since the remaining films B40, B60, B120 and C40, C60, C120 simply follow the trend in Fig. 6(a).

Figure 6(b) plots the average $2 \theta$ values of the experimentally obtained $\alpha$-(110) reflection for the out-of-plane scans given in black in Fig. 6(a) and for the in-plane scans (XRD patterns not shown) as a function of film thickness for films B20-B180. Fig. 6(b) also provides the $2 \theta$ values for unstrained $\alpha-(110)$ reflection given by Noyan and $\mathrm{Shaw}^{30}$ As can be seen in the figure, for both the as-deposited and annealed films, the shifts in the peak positions relative to the unstrained state occur in opposite directions for the out-ofplane versus the in-plane scans. Therefore, if the film is in compression in-plane, it is in tension out-of-plane, consistent with the Poisson effect.

Further examination of Fig. 6(a) shows that the $\alpha-(110)$ peaks of the as-deposited films are asymmetric, in agreement with Noyan and Shaw. ${ }^{30}$ The Bragg angle for the unstrained $\alpha$-(110) reflection is shown as a vertical line in the XRD scans in Fig. 6(a). One of the deconvoluted peaks has a higher $2 \theta$ (thus a smaller $d_{110}$ along the film normal) than that for the unstrained state, whereas the other deconvoluted peak has a lower $2 \theta$ (thus larger $d_{110}$ along the film normal) than that for the unstrained state. The percentage shifts in $d_{110}$ along the film normal relative to the unstrained value are shown in Fig. 6(c). After annealing at $850{ }^{\circ} \mathrm{C}$, only a single, symmetric $\alpha-(110)$ peak was observed for the 12 films B20-B180 and C20-C180. The deviation of the interplanar spacing for this peak from the unstrained value was less than $0.15 \%$. Although the higher thermal expansion coefficient of W $\left(4.3 \times 10^{-6} /{ }^{\circ} \mathrm{C}\right)$ compared with that of the $\mathrm{SiO}_{2}\left(0.4 \times 10^{-6} /{ }^{\circ} \mathrm{C}\right) / \mathrm{Si}\left(3.08 \times 10^{-6} /{ }^{\circ} \mathrm{C}\right)$ substrate should give rise to tensile stress in the $\mathrm{W}$ layer upon cooling following annealing, Fig. 6(b) evidences a more complex picture as a result of the combined effect of atomic peening (compressive in the as-deposited state) and the $\beta$ to $\alpha$ transformation (tensile in the as-deposited state). Annealing at $400^{\circ} \mathrm{C}$, however, resulted in no observable change in the XRD peak shapes and positions relative to the as-deposited films. (data not shown).

Figures 6(a) and 6(c) indicate that, in agreement with Noyan and Shaw ${ }^{30}$ an inhomogeneous stress state exists in the as-deposited films studied here, wherein a fraction of the $\alpha-\mathrm{W}$ is under a compressive in-plane stress (smaller $2 \theta$ ) and the remaining fraction is under a tensile in-plane stress (larger 20). The two groups of films B20-B180 and C20C180 showed similar thickness dependence for the compressive and tensile in-plane stresses as evidenced by the change (a)
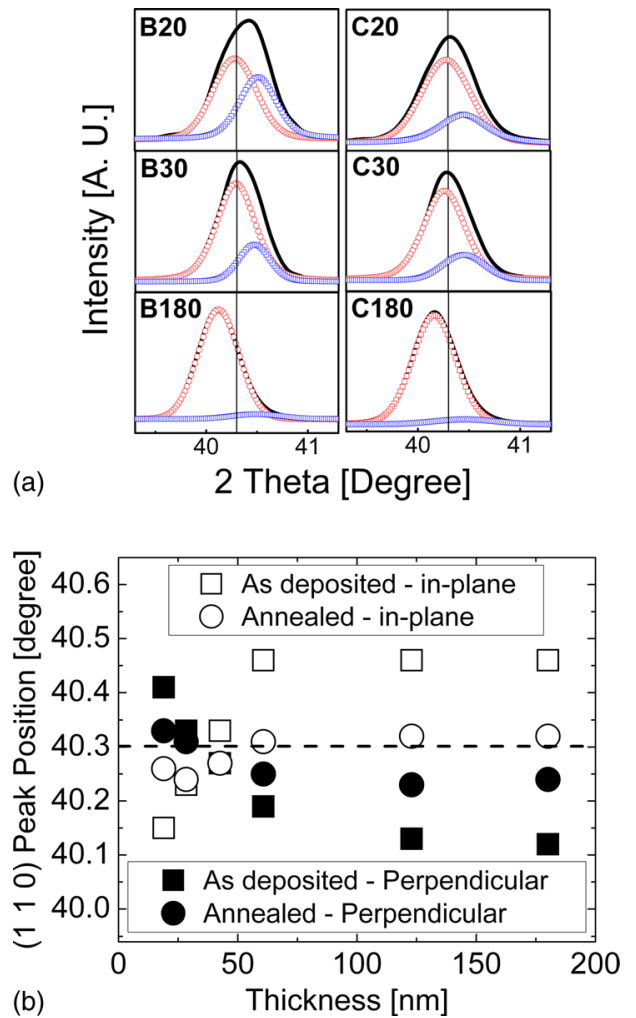
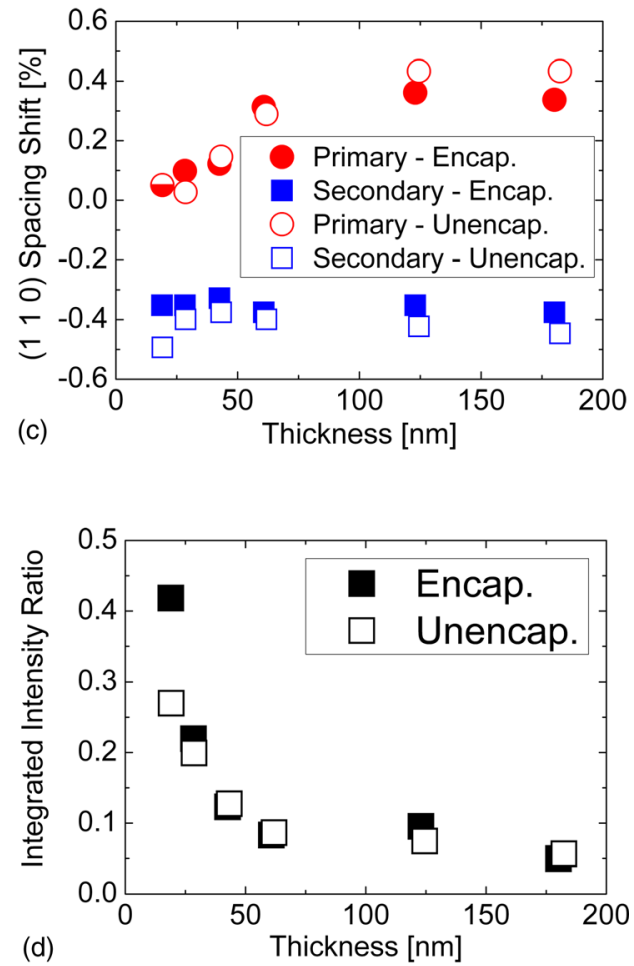

FIG. 6. (Color online) (a) Out-of-plane $\alpha$-(110) XRD peaks of films B20, B30, B180, C20, C30, and C180 in the as-deposited state. The experimentally observed peaks (black solid lines) were deconvoluted into the primary $\alpha$-W (red open circles) and secondary $\alpha$-W (blue open squares) peaks. (b) The average (110) peak positions for films B20-B180, obtained from in-plane and out-of-plane XRD $\theta-2 \theta$ scans are shown as a function of thickness. The dotted horizontal line represents the expected peak position for unstrained $\alpha$-W using the value of lattice parameter given in Ref. 30. (c) The difference in the (110) interplanar spacing of primary $\alpha-\mathrm{W}$ (circles) and secondary $\alpha-\mathrm{W}$ (squares) along the film normal direction relative to the unstrained interplanar spacing is shown as a function of film thickness. The half-closed circle represents the two identical data points (d) The integrated intensity ratios (secondary $\alpha$-W)/(primary $\alpha$ $\mathrm{W}+$ secondary $\alpha-\mathrm{W})$ for the unencapsulated films (B20-B180) and encapsulated films (C20-C180) from (a) are shown as a function of thickness. 
in the $d_{110}$ perpendicular to the film seen in Fig. 6(c). The compressive in-plane stress increased in magnitude as film thickness increased to $60 \mathrm{~nm}$, and then became independent of thickness, with a maximum observed shift in $d_{110}$ of approximately $+0.4 \%$. In contrast, the in-plane tensile stress showed no thickness dependence, as evidenced by a nearly constant shift in $d_{110}$ of $-0.4 \%$.

Noyan and Shaw ${ }^{30}$ termed the $\alpha-\mathrm{W}$ under compressive inplane stress "primary $\alpha$," and the $\alpha$-W under tensile in-plane stress "secondary $\alpha-\mathrm{W}$." Primary $\alpha-\mathrm{W}$ forms by deposition of W directly in the A2 (bcc) structure, with the compressive in-plane stress resulting from atomic "peening" effects. An explanation for atomic peening is that bombardment by energetic sputtered atoms and neutral sputtering gas atoms causes the incorporation of excess atoms in the film, e.g., along grain boundaries. Since the film is constrained by the substrate, these excess atoms result in a compressive stress, in cases where adatom mobility is too low to allow relaxation during growth. ${ }^{37-39}$

Secondary $\alpha-\mathrm{W}$ forms by transformation of A15 $\beta$-W to A2 $\alpha-\mathrm{W}$ as film deposition proceeds. The tensile in-plane stress in secondary $\alpha-\mathrm{W}$ (associated with the compressive out-of-plane strain, i.e., smaller $d_{110}$ along the film nor$\mathrm{mal}$ ) is a consequence of the volumetric reduction accompanying the transformation of $\beta$-W immediately adjacent to the substrate. ${ }^{30}$ Hence, secondary $\alpha-\mathrm{W}$ acts as an indicator for the presence of $\beta-\mathrm{W}$ in the early stages of film deposition.

The integrated intensity of the secondary $\alpha-\mathrm{W}$ (110) peak relative to the total integrated peak intensity for this peak allows a rough estimate to be made of the amount of $\beta$-W in the films and the thickness during deposition at which the transformation of $\beta$-W to (secondary) $\alpha-\mathrm{W}$ can be considered as complete. The integrated intensity ratios for as-deposited films B20-B180 and C20-C180 are given in Fig. 6(d). Comparison of the results for the encapsulated and unencapsulated films indicates more secondary $\alpha-\mathrm{W}$ and thus $\beta-\mathrm{W}$ in the former. The $\theta / 2 \theta$ scans can be used for this estimate, since the films are largely untextured.

For the unencapsulated films $\mathrm{C} 20-\mathrm{C} 180$, if the thickness of secondary $\alpha-\mathrm{W}$ is assumed to be $5 \mathrm{~nm}$, then the ratio of this thickness to the total film thickness gives values of 0.27 (C20), 0.18 (C30), 0.12 (C40), 0.08 (C60), 0.04 (C120), and 0.03 (C180). These values are in reasonably good agreement with the integrated intensity ratios for these films given in Fig. 6(d), particularly for the thinner films C20-C60. From this, we conclude that the thickness at which the transformation from $\beta$-W to $\alpha-\mathrm{W}$ is complete for unencapsulated films is $\sim 5 \mathrm{~nm}$.

For the encapsulated films B20-B180, the intensity ratios in Fig. 6(d) indicate a thickness for secondary $\alpha-\mathrm{W}$ greater than $5 \mathrm{~nm}$, but less than $10 \mathrm{~nm}$, in good agreement with the electron and XRD patterns in Figs. 2 and 3, wherein film B5 was a mixture of $\alpha-\mathrm{W}$ and $\beta$-W and film B10 was single phase $\alpha-\mathrm{W}$. Hence, the thickness at which the transformation from $\beta-\mathrm{W}$ to (secondary) $\alpha-\mathrm{W}$ can be considered complete for the encapsulated films is between 5 and $10 \mathrm{~nm}$.

\section{Resistivity}

Film resistivities in the as-deposited state and after annealing at $850^{\circ} \mathrm{C}$ for $2 \mathrm{~h}$ are listed in Table I and are plotted as a function of thickness for films B5-B180 and films C5-C180 in Fig. 7. The resistivities are as high as $137.8 \mu \Omega$ $\mathrm{cm}$ in the as-deposited state and as low as $8.6 \mu \Omega \mathrm{cm}$ in the annealed state. The latter value is, to our knowledge, the lowest reported value for sputtered W films and is comparable to the reported resistivities for chemical vapor deposited (CVD) $\mathrm{W}$ films. ${ }^{14,22}$ However, all the resistivity values listed in Table I are higher than the bulk resistivity of $5.5 \mu \Omega \mathrm{cm}$ at $301 \mathrm{~K}$ for single phase A2 $\alpha$-W.

The possible reasons for the higher resistivity values include: (1) the presence of $\mathrm{A} 15 \beta$ - $\mathrm{W}$, (2) the incorporation of impurities, and (3) the presence of surfaces and grain boundaries. The presence of $\beta-\mathrm{W}$ (Figs. 1-3) is clearly the major contributor to the resistivity values of $>100 \mu \Omega \mathrm{cm}$ for as-deposited films A20 and B5. Impurities inherited from the sputtering target and additionally incorporated during film fabrication, particularly from oxygen bearing gases residual in the chamber or introduced during deposition of the lower $\mathrm{SiO}_{2}$ layer, also contribute to the higher film resistivities compared to the bulk resistivity. This contribution is either direct via impurity scattering or indirect via the stabilization of $\beta-\mathrm{W}$. As an example of the latter, the resistivity of as-deposited film A20 for which the base pressure of the chamber was $5 \times 10^{-7}$ Torr and the film was $\beta$-W was $137.8 \mu \Omega \mathrm{cm}$, whereas the resistivity of as-deposited film B20 for which the base pressure was $2 \times 10^{-9}$ Torr and the film was $\alpha-\mathrm{W}$ was significantly lower at $18.6 \mu \Omega \mathrm{cm}$.

With regard to the direct contribution of impurities incorporated during film fabrication, the deposition of the lower $\mathrm{SiO}_{2}$ layer should make a greater contribution compared with that of chamber base pressure, because of the higher partial pressure of oxygen-containing species introduced during $\mathrm{SiO}_{2}$ deposition. This assertion is supported by comparing the resistivities of the $850^{\circ} \mathrm{C}, 2 \mathrm{~h}$ annealed films, which are single phase $\alpha-\mathrm{W}$ after the annealing treatment.

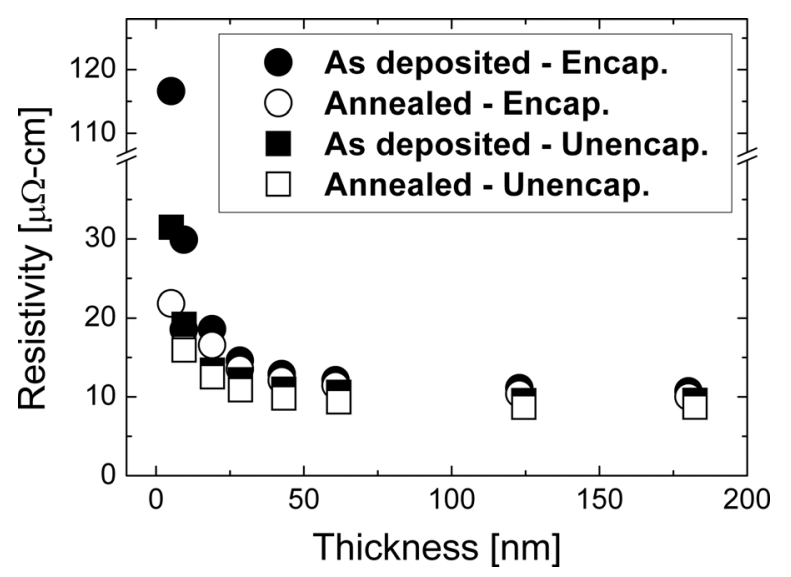

FIG. 7. Resistivity as a function of thickness for films B5-B180 (circles) and C5-C180 (squares) in the as-deposited state (closed symbols) and after annealing at $850^{\circ} \mathrm{C}$ for $2 \mathrm{~h}$ (open symbols). The resistivity of film C5 after the annealing treatment is not shown due to film agglomeration in the absence of the encapsulant. 
For the nominally 20-nm-thick films, resistivities of annealed films A20, B20, and C20 are 17.0, 16.5, and 12.5 $\mu \Omega \mathrm{cm}$, respectively. The nominally 180 -nm-thick films show a similar trend in resistivity, but the resistivity differences are smaller. This is seen is by comparing the resistivities of films A180, B180, and C180 at 10.2, 10.0, and 8.6 $\mu \Omega \mathrm{cm}$, respectively.

When as-deposited and annealed film resistivities are compared, it is seen that annealing results in a reduction of resistivity for all films. An exception is film C5, which agglomerates upon annealing due to the absence of the top encapsulation layer. For films A20 and B5, the transformation of $\beta-\mathrm{W}$ to (secondary) $\alpha-\mathrm{W}$ is a major source of the reduction in resistivity following annealing, as seen in Fig. 7 and Table I. It was also seen that annealing results in the relaxation of the in-plane compressive stress in the films, presumably because of removal of the excess atoms in the film. ${ }^{37,39}$ If the removal of these atoms and the associated reduction in film stress is a contributor to the reduction in resistivity, then it indicates the sensitivity of electron scattering in $\mathrm{W}$ to any deviations from the relaxed equilibrium state. Figure 7 also shows that the resistivity increase becomes increasingly greater as film thickness reduces, whereas a nearly constant resistivity was achieved for film thicknesses above $120 \mathrm{~nm}$. This observation will be discussed in more detail in Sec. III E.

\section{E. Discussion}

The formation of A15 $\beta$-W has been shown to be sensitive to a variety of film preparation parameters ${ }^{14,24,40-43}$ and prior to the work of Rossnagel et al., ${ }^{14}$ it was believed that the transformation from $\beta$-W to $\alpha-\mathrm{W}$ would occur at a fixed film thickness (critical thickness) reported as $\sim 50 \mathrm{~nm}$ by Vink et $a l .{ }^{43}$ Rossnagel et al. ${ }^{14}$ argued that the transformation from $\beta$-W to $\alpha-\mathrm{W}$ is simply a result of heating associated with the sputtering process itself-thicker films are at higher temperature simply due to longer deposition time for a given deposition rate-and hence there is no fixed critical thickness beyond which deposition occurs in the $\alpha-\mathrm{W}$ structure, ${ }^{14}$ They calculated a temperature $\geq 125^{\circ} \mathrm{C}$ for a deposited thickness of $45 \mathrm{~nm}$.

Our findings regarding the formation of $\beta$-W are in agreement with the conclusion of Rossnagel et al. ${ }^{14}$ in that the thickness at which $\alpha$-W forms during deposition is not fixed. This thickness does, however, depend on the deposition conditions other than substrate temperature. For example, for group A, film A20 is $\beta-\mathrm{W}$ with a resistivity of $137.8 \mu \Omega \mathrm{cm}$. In contrast, for group $\mathrm{B}, \beta-\mathrm{W}$ is only found in the nominally 5 -nm-thick film as a mixture of $\alpha-\mathrm{W}$ and $\beta$-W, with a resultant film resistivity of $116.6 \mu \Omega \mathrm{cm}$. Fully $\alpha-\mathrm{W}$ was achieved at a thickness of $10 \mathrm{~nm}$ (film B10), resulting in a film resistivity of $29.8 \mu \Omega \mathrm{cm}$ in the as-deposited state. For group C, even the thinnest film (C5) was fully $\alpha-\mathrm{W}$ with a resistivity of $31.4 \mu \Omega \mathrm{cm}$ in the as-deposited state.

As noted in Sec. I, obtaining a resistivity equal to bulk resistivity of $5.3 \mu \Omega \mathrm{cm}$ at $293 \mathrm{~K}(5.5 \mu \Omega \mathrm{cm}$ at $301 \mathrm{~K})$ for polycrystalline $\mathrm{W}$ films has proved challenging. Table II presents a summary of thick-film resistivity values for poly- crystalline $\mathrm{W}$ films made by sputter deposition, CVD, and atomic layer deposition (ALD), taken from the literature. As can be seen, the reported film resistivities are significantly higher than the reported bulk resistivity for all three deposition methods. For CVD and ALD, this is the result of incorporation of fluorine from the $\mathrm{W}$ source gas, $\mathrm{WF}_{6}$, whereas for sputter-deposited films this is generally attributed to the presence of $\beta-\mathrm{W}$, and the incorporation of impurities. ${ }^{14}$ Therefore, in order to obtain the bulk resistivity, it is imperative to minimize the impurity incorporation and eliminate $\beta$-W.

The resistivity of $8.6 \mu \Omega \mathrm{cm}$ for films $\mathrm{C} 120$ and $\mathrm{C} 180$ obtained after annealing is less than the previously reported values for sputtered $\mathrm{W}$ films and is comparable to the resistivity for CVD W films. Since the target purity for our film is only $99.95 \%$, we believe that by using higher purity targets and further reducing impurities during film fabrication, resistivities comparable to the bulk resistivity will be achievable.

For the annealed films in groups $\mathrm{B}$ and $\mathrm{C}$, resistivity increased as film thickness decreased below $120 \mathrm{~nm}$ (Fig. 7). This increase can result from a number of scattering mechanisms. Two of the most important are electron scattering at film surfaces and at grain boundaries. ${ }^{44-46}$ In order for grain boundary scattering to be the major contributor to the resistivity increase (Fig. 7), the grain size of the films would have had to decrease as film thickness decreased. Table I shows this is not the case for group B, and approximate constancy of grain size with thickness is expected for group $\mathrm{C}$, since, as discussed in Sec. III B, it is hypothesized that the grain size of $\alpha-\mathrm{W}$ is determined by the $\beta$ to $\alpha$ transformation (Fig. 2) in the early stages of deposition.

Having ruled out grain boundary scattering as the dominant mechanism, surface scattering is expected to be the major contributor to the resistivity increase with decreasing film thickness seen in Fig. 7. As film thickness decreases, conduction electrons more frequently encounter the surfaces and lose the momentum gained from the electric field if diffusely scattered. However, quantitative analysis of the surface scattering contribution in film groups $\mathrm{B}$ and $\mathrm{C}$ is hindered by the lack of a reliable value for the EMFP in $\mathrm{W} .{ }^{14,15}$ Work is under way in our laboratory to determine the EMPF. The behavior of the $\mathrm{W}$ films is to be contrasted with $\mathrm{Cu}$ films, where grain boundary scattering is found to

TABLE II. Summary of deposition methods and the corresponding thickfilm resistivities for polycrystalline $\mathrm{W}$ films reported in the literature.

\begin{tabular}{lcc}
\hline \hline Deposition method $^{\mathrm{a}}$ & Thick-film resistivity $(\mu \Omega \mathrm{cm})$ & Reference \\
\hline SD & 60 & 23 \\
SD & 20 & 24 \\
SD & 12 & 14 \\
SD & 40 & 25 \\
SD & 10.3 & 26 \\
CVD & 8 & 22 \\
CVD & 13 & 27 \\
CVD & 8.7 & 28 \\
ALD & 115 & 29 \\
\hline \hline
\end{tabular}

${ }^{\mathrm{a}} \mathrm{SD}, \mathrm{CVD}$, and ALD stand for sputter deposition, chemical vapor deposition, and atomic layer deposition, respectively. 
provide the strongest contribution to the resistivity increase, with a weaker role observed for surface scattering. ${ }^{3,4}$

\section{SUMMARY AND CONCLUSIONS}

For the films studied, $x$-ray and electron diffraction showed that by reducing the base pressure prior to film deposition from $5 \times 10^{-7}$ to $2 \times 10^{-9}$ Torr and by eliminating the encapsulating $\mathrm{SiO}_{2}$ layers, single phase, polycrystalline $\alpha-\mathrm{W}$ could be achieved in as-deposited films as thin as $5 \mathrm{~nm}$. From the examination of the grain structure of the films in the as-deposited state and measurement of grain size for a subset of the $850{ }^{\circ} \mathrm{C}, 2 \mathrm{~h}$ annealed films, it was concluded that once the $\beta$ to $\alpha$ transformation is complete, the low mobility of grain boundaries causes the grain size to remain essentially unchanged as the film thickens during deposition or is annealed after deposition. For the $\alpha-\mathrm{W}$ films in the asdeposited state, the stress was found to be inhomogeneous, with primary $\alpha-\mathrm{W}$ (deposited directly in the A2 structure) under compressive in-plane stress, and secondary $\alpha-\mathrm{W}$ (formed by transformation of A15 $\beta$-W that is immediately adjacent to the substrate to $\mathrm{A} 2 \alpha-\mathrm{W}$ ) under tensile in-plane stress. Annealing resulted in both relaxation of the stress and a reduction of resistivity for all films, except the thinnest unencapsulated film $\mathrm{C} 5$, that agglomerated during annealing.

The thick-film resistivity of $8.6 \mu \Omega \mathrm{cm}$ for film C180 obtained after annealing is less than the previously reported values for sputtered W films and is comparable to the resistivity for CVD W films. Future studies will focus on process modifications (e.g., the use of higher purity targets) to achieve thick-film resistivities that are equal to the reported bulk resistivity of $5.3 \mu \Omega \mathrm{cm}$ for polycrystalline films. The resistivity of the annealed films was found to increase with decreasing thickness below $120 \mathrm{~nm}$. Hence, future studies will also address the quantitative separation of the contributions of the various scattering sources to resistivity. In short, additional studies of the resistivity of $\mathrm{W}$ films are needed before $\mathrm{W}$ can be considered as a viable alternative to $\mathrm{Cu}$ as an interconnect metal.

\section{ACKNOWLEDGMENTS}

Financial support from the Alessandro and Piermaria Reggiori Fellowship, the Bertucci Graduate Fellowship, the Department of Materials Science and Engineering, Intel Corporation, the Semiconductor Research Corporation Task 1292.008 and Task 2121.001, and partial support from the MRSEC program of the NSF under DMR-0520425 are gratefully acknowledged. Portions of this research were carried out at the SSRL, a national user facility operated by Stanford University on behalf of the U.S. Department of Energy, Office of Basic Energy Sciences.

${ }^{1}$ F. Chen and D. Gardner, IEEE Electron Device Lett. 19, 508 (1998).

${ }^{2}$ T. Sun, B. Yao, A. P. Warren, V. Kumar, S. Roberts, K. Barmak, and K. R. Coffey, J. Vac. Sci. Technol.A 26, 605 (2008).

${ }^{3}$ T. Sun, B. Yao, A. P. Warren, K. Barmak, M. F. Toney, R. E. Peale, and K. R. Coffey, Phys. Rev.B 79, 041402 (2009).

${ }^{4}$ T. Sun, B. Yao, A. P. Warren, K. Barmak, M. F. Toney, R. E. Peale, and K. R. Coffey, Phys. Rev.B 81, 155454 (2010).
${ }^{5}$ http://www.itrs.net/links/2007itrs/2007_Chapters/2007_Interconnect. pdf.

${ }^{6}$ C.-K. Hu, R. Rosenberg, and K. Y. Lee, Appl. Phys. Lett. 74, 2945 (1999).

${ }^{7}$ D. N. Bhate, A. Kumar, and A. F. Bower, J. Appl. Phys. 87, 1712 (2000) .

${ }^{8}$ J. R. Lloyd, M. W. Lane, E. G. Liniger, C.-K. Hu, T. M. Shaw, and R. Rosenberg, IEEE Transs. Device Mater. Reliab. 5, 113 (2005).

${ }^{9}$ Y. Wul, J. Xiang, C. Yang, W. Lu, and C. M. Lieber, Nature (London) 430, 61 (2004).

${ }^{10}$ A. Nieuwoudt and Y. Massoud, IEEE Transs.Electron Devices 53, 2460 (2006).

${ }^{11}$ P. Avouris, Z. Chen, and V. Perebeinos, Nat. Nanotechnol. 2, 605 (2007).

${ }^{12}$ K. C. Cadien, M. R. Reshotko, B. A. Block, A. M. Bowen, D. L. Kencke, and P. Davids, Proc. SPIE 5730, 133 (2005).

${ }^{13} \mathrm{http}$ // /www.itrs.net/Links/2009ITRS/2009Chapters_2009Tables/2009_ Interconnect.pdf.

${ }^{14}$ S. M. Rossnagel, I. C. Noyan, and C. Cabral, Jr., J. Vac. Sci. Technol.B 20, 2047 (2002).

${ }^{15}$ For $\mathrm{W}$, the value of $2 \mathrm{~nm}$ is calculated as the product of scattering time (from bulk resistivity) and Fermi velocity of $4 \times 10^{5} \mathrm{~m} / \mathrm{s}$, as determined by Janossy and Kollar from measurement of electron spin waves in W [A. A. Janossy and J. Kollar, J. Phys.F: Met. Phys. 8, 2429 (1978)], and consistent with the separate calculation by Kollar of $5 \times 10^{5} \mathrm{~m} / \mathrm{s}$.

${ }^{16}$ http://www.hbcpnetbase.com/.

${ }^{17} \mathrm{This}$ value is obtained by assuming an EMPF of $2 \mathrm{~nm}$, and a Matthiessen's rule summation of surface and grain boundary scattering with their associated parameters taken from Ref. 4 for both $\mathrm{Cu}$ and $\mathrm{W}$. The values of $\mathrm{TaN} / \mathrm{Ta}$ adhesion-diffusion barrier thicknesses used in the calculation of $\mathrm{Cu}$ line resistivities are taken from ITRS 2007.

${ }^{18}$ C. S. Hau-Riege, Microelectron. Reliab. 44, 195 (2004).

${ }^{19} \mathrm{D}$. Edelstein et al., Proceedings of the IEEE Interconnect Technology Conference, Burlingame, CA, USA, 6 June, 2001 (IEEE, New York, 2001).

${ }^{20}$ The barrier metal thickness for $\mathrm{Cu}$ metal 1 wiring is expected to be $2.9 \mathrm{~nm}$ for 2011 according to ITRS 2007.

${ }^{21}$ P. J. Ireland, Thin Solid Films 304, 1 (1997).

${ }^{22}$ I. P. Ivanov, I. Sen, and P. Keswick, J. Vac. Sci. Technol.B 24, 523 (2006).

${ }^{23}$ J. Ligot, S. Benayoun, and J. J. Hantzpergue, J. Vac. Sci. Technol.A 19, 798 (2001)

${ }^{24}$ G. S. Chen, H. S. Tian, C. K. Lin, G.-S. Chen, and H. Y. Lee, J. Vac. Sci. Technol.A 22, 281 (2004).

${ }^{25}$ M. J.O 'Keefe and J. T. Grant, J. Appl. Phys. 79, 9134 (1996).

${ }^{26}$ A. S. Kao, C. Hwang, V. J. Novotny, V. R. Deline, and G. L. Gorman, J. Vac. Sci. Technol.A 7, 2966 (1989).

${ }^{27}$ C. Kim, I. Rho, S. Kim, I. Han, H. Kang, S. Ryu, and H. Kim, J. Electrochem. Soc. 156, H685 (2009).

${ }^{28}$ W. Steinhögl, G. Steinlesberger, M. Perrin, G. Scheinbacher, G. Schindler, M. Traving, and M. Engelhardt, Microelectronic Eng. 82, 266 (2006).

${ }^{29}$ S. Kim et al., Electrochem.Solid-State Lett. 8, C155 (2005).

${ }^{30}$ I. C. Noyan and T. M. Shaw, J. Appl. Phys. 82, 4300 (1997).

${ }^{31}$ A. D. Darbal, K. Barmak, N. T. Nuhfer, K. R. Coffey, B. Yao, X. Liu, D. Choi, and T. Sun, "Grain size quantification of nanocrystalline materials in the TEM using conical dark field imaging," Micron (submitted).

${ }^{32}$ D. J. Dingley, Microchim. Acta 155, 19 (2006).

${ }^{33}$ D. T. Carpenter, J. M. Rickman, and K. Barmak, J. Appl. Phys. 84, 5843 (1998).

${ }^{34}$ L. J. Van der Pauw, Philips Res. Rep. 13, 1 (1958).

${ }^{35}$ C. V. Thompson, Annu. Rev. Mater. Sci. 30, 159 (2000).

${ }^{36}$ J. A. Thornton, Annu. Rev. Mater. Sci. 7, 239 (1977).

${ }^{37}$ F. M. d'Heurle and J. M. E. Harper, Thin Solid Films 171, 81 (1989).

${ }^{38}$ J. A. Thornton and D. W. Hoffman, Thin Solid Films 171, 5 (1989).

${ }^{39}$ E. Chason, B. W. Sheldon, and L. B. Freund, Phys. Rev. Lett. 88, 156103 (2002).

${ }^{40}$ I. A. Weerasekera, S. I. Shah, D. V. Baxter, and K. M. Unruh, Appl. Phys. Lett. 64, 3231 (1994).

${ }^{41}$ A. M. Haghiri-Gosnet, F. R. Ladan, C. Mayeus, and H. Launois, J. Vac. Sci. Technol.A 7, 2663 (1989).

${ }^{42}$ T. Karabacak, A. Mallikarjunan, J. P. Singh, D. Ye, G. Wang, and T. Lu, Appl. Phys. Lett. 83, 3096 (2003).

${ }^{43}$ T. J. Vink, W. Walrave, J. C. L. Daams, A. G. Dirks, M. A. J. Somers, and K. J. A. Van der Aker, J. Appl. Phys. 74, 988 (1993).

${ }^{44}$ K. Fuchs, Proc. Cambridge Philos. Soc. 34, 100 (1938).

${ }^{45}$ E. H. Sondheimer, Adv. Phys. 1, 1 (1952).

${ }^{46}$ A. F. Mayadas and M. Shatzkes, Phys. Rev.B 1, 1382 (1970). 